\title{
EFFECT OF DRIVING EXPERIENCE ON CHANGE DETECTION BASED ON TARGET RELEVANCE AND SIZE
}

\author{
Alexandra S. Mueller ${ }^{1} \&$ Lana M. Trick $^{2}$ \\ ${ }^{1}$ Western University, London, Ontario, Canada \\ ${ }^{2}$ University of Guelph, Guelph, Ontario, Canada \\ E-mail: amueller@alumni.uoguelph.ca
}

\begin{abstract}
Summary: Earlier studies that investigated the effects of driving experience and target safety relevance on change detection have produced conflicting results. Using a flicker change detection task to investigate the effect of driving experience on the ability to detect changes in objects that vary in safety relevance and size, the present study attempted to clarify some controversies by addressing three important methodological issues. The data showed that experienced drivers exhibited more efficient selection strategies than novice drivers and thus may have more spare resources to analyze less relevant objects in the driving environment. Selection strategies for relevant information appear to be sensitive to object size whereas selection of irrelevant information is downgraded comparatively and unaffected by size. Findings are discussed as they relate to theoretical and practical implications.
\end{abstract}

\section{INTRODUCTION}

A widespread theory about learning to drive is that motorists need to develop knowledge and expectations about information that is relevant to avoid collisions, and that experience increases the probability of drivers selecting safety-related information over safety-unrelated information (Trick, Enns, Mills \& Vavrik, 2004). One of the most consistent effects in the driving-related change detection literature is that relevant information (such as road users) tends to be detected faster and more accurately than irrelevant information (e.g., Velichkovsky, Dornhoefer, Kopf, Helmert \& Joos, 2002). However, the few studies that have examined the effects of driving expertise and target safety relevance have produced conflicting results. Galpin, Underwood, and Crundall (2009) did not find an effect of driving experience, whereas Groff and Chaparro (2003) found that very experienced drivers detected changes in relevant information faster than less experienced drivers. In contrast, Tanaka, Ikeda, Qingwei, and Ishiguchi (2009) found that all drivers detected relevant changes similarly although experienced drivers were slower than novice drivers at detecting irrelevant changes.

Three important methodological differences between these studies may help to explain the discrepancies in their findings. First, most of the earlier studies focused on response time while ignoring error rate and it is impossible to know whether the participants in different studies were equally successful at identifying the exact origin of the change. It is also difficult to compare studies that do not report the same dependent variables. Therefore, in this study we used a flicker change detection task (Rensink, O’Regan \& Clark, 1997) and measured both response time and error rate. The second issue concerns target location. Pringle, Irwin, Kramer, and Atchley (2001) showed that centrally located information tends to be detected better than peripheral information. It is hard to control target location in naturalistic driving scenes and sometimes it is difficult to 
determine whether it was the targets' safety relevance or location that produced a difference in performance. Therefore, in this study we created road traffic scenes showing multiple vantage points from a driver's point of view to control the location of targets without compromising their relevance to the driving scene. The vantage points included straight roads with straight approaches as well as curved roads and angled approaches. The third problematic issue was the age of participants. Age not only affects the ability to detect changes in road traffic scenes (Batchelder, Rizzo, Vanderleest \& Vecera, 2003), but also brings along some driving-unrelated factors such as maturity. Several of the previous studies had significant age differences between their experienced and novice driver groups, which might explain some of the disagreement among the results. In order to investigate the effects of driving experience while minimizing the influence of age this study was restricted to participants under the age of 30 .

Although information can stand out from the environment based on its relevance to the context of the task, it can also be conspicuous to the observer based on its physical characteristics or visual conspicuity (Engel, 1977). To date, little research has investigated whether learning to drive involves learning to prioritize visual information differently based on safety relevance. Many factors affect visual conspicuity but, as a start, we chose object size. Road users are of primary importance to a motorist's ability to drive safely, yet there are different types of road users and they vary in size. Small road users such as cyclists, pedestrians and motorcyclists may be especially at risk for collision because they are less visually conspicuous than passenger vehicles (Thornley, Woodward, Langley, Ameratunga \& Rodgers, 2008). Hurt, Ouellet, and Thom (1981) suggested that less visually conspicuous road users might be more conspicuous to experienced drivers than to novice drivers. If this is the case, experienced drivers may have an advantage because they are more familiar with driving situations in which small road users are present and have more accurate expectations about them. We hypothesized that experienced drivers allocate more resources to smaller road users and should detect them similarly to large road users. In contrast, novice drivers allocate their resources based on visual conspicuity and should be worse at detecting smaller road users than larger ones. In terms of irrelevant information, we expected that size should have the same effect on all drivers regardless of experience, and all should be worse at detecting changes in small objects than in large ones.

\section{METHOD}

\section{Participants}

Participants were divided into two groups based on experience. The more experienced drivers ( $n$ $=19,12$ males) were on average 23 years old $(S D=1.92)$, had an average of 7 years of driving experience $(S D=1.47)$, and held a valid full driver's license. Novice drivers ( $n=19,4$ males) were on average 19 years old $(S D=1.89)$, had an average of half a year of driving experience $(S D=0.31)$, and held at minimum a valid entry-level driver's license. Participants were recruited from the university undergraduate participant pool and public advertisements, and were paid one credit towards the research component of their course grade or \$10.00 for their participation.

\section{Apparatus and Stimuli}

Road traffic scenes were presented using VScope ${ }^{\mathrm{TM}}$ software (Enns \& Rensink, 1992) on a 15inch display Macintosh G4 PowerBook laptop and subtended $17.34^{\circ}$ wide by $12.93^{\circ}$ when 
viewed from a distance of $60 \mathrm{~cm}$ (see Figure 1). Targets in every condition were counterbalanced between center and periphery. Central targets were located within $1.81^{\circ}$ from the centre of the display, and peripheral targets were located $5.34^{\circ}$ or more away from the centre. Target position in every condition (as well as center and periphery) was counterbalanced between the left and right side of the display. Large relevant targets were sedans, pickup trucks and sports utility vehicles (SUVs), and small relevant targets were cyclists, pedestrians and motorcyclists. Large irrelevant targets were dumpsters, sheds, and construction arrow trailers, and small irrelevant targets were mailboxes, garbage bins and phone booths. Irrelevant targets were approximately equivalent in size to relevant targets. There were 12 replications for each condition yielding a total of 48 experimental trials. Every condition contained four presentations of each target type.

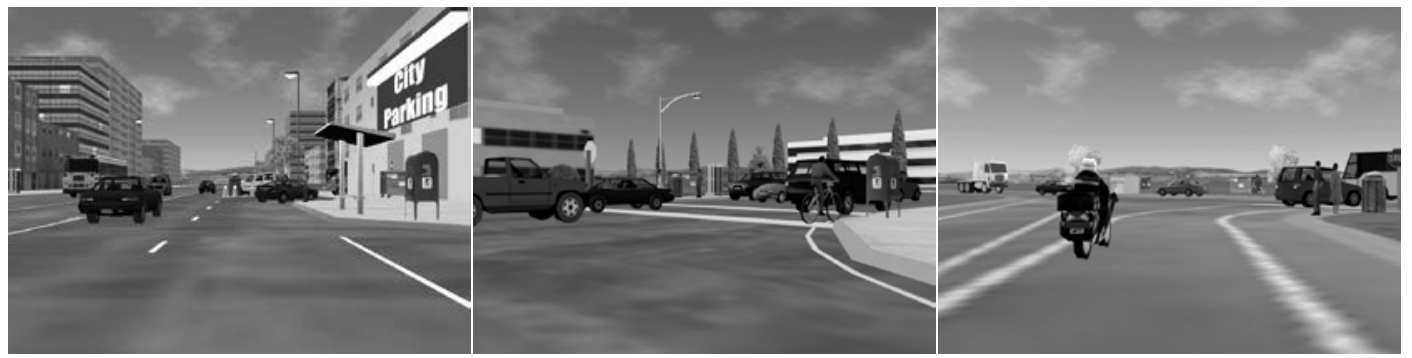

Figure 1. Examples of road traffic scenes from multiple driving-related vantage points

\section{Procedure}

Each trial presented two images that were almost identical, except that the second image had the addition of a novel object (target) not present in the first. Participants were instructed to identify what changed (i.e., the target) between successive images. There was one change per trial. Upon pressing the spacebar (with their dominant hand), participants were shown a white blank display for $0.5 \mathrm{~s}$, followed by a fixation cross in the center of the white display. The fixation cross remained onscreen for $1 \mathrm{~s}$ and was followed by a blank display for $0.1 \mathrm{~s}$, after which the first image of the pair appeared for $1 \mathrm{~s}$. A blank display followed for $0.1 \mathrm{~s}$ and then the second image was presented for $1 \mathrm{~s}$, followed by another blank display for $0.1 \mathrm{~s}$. This cycle was repeated starting from the first image until the participant pressed the spacebar (to indicate that they saw the change) or 300 repetitions had been shown. None of the participants required more than 300 repetitions. Response time was measured from the initial presentation of the second image of the pair (which contained the change) to the spacebar press. After the participant pressed the spacebar a fixation cross was presented for $1 \mathrm{~s}$, followed by a blank display for $0.1 \mathrm{~s}$. The second image of the pair was presented for $3 \mathrm{~s}$ during which the participant told the experimenter what the change was. The experimenter recorded the response and, if participants made an incorrect identification, the experimenter corrected them. Trial presentation was self-paced and initiated by a spacebar press. Trials were randomly ordered, and six practice trials preceded 48 experimental trials. Upon completion participants completed a questionnaire about driving habits and other demographic information.

\section{RESULTS}

Error rate and response time were analyzed independently by means of 2(driving experience) $\mathrm{x}$ 2(safety relevance) x 2(size) split-plot factorial ANOVAs. Response time data were non-normal 
and thus a $\log _{10}$ transformation was performed prior to analysis. All ANOVAs used GreenhouseGeisser corrections for violations of the sphericity assumption. Holm's sequential Bonferronicorrected pairwise comparisons at the .05 significance level were used for post-hoc test of means. Given the gender and age imbalance between groups, additional ANCOVAs with age and gender as covariates were performed for error rate and response time. Occasionally, participants pressed the spacebar prematurely before they had seen the second display (and thus the change), which led to negative response times. These trials were removed from analysis $(0.4 \%$ in total). No participant had more than one of these mistakes per condition. Only trials with correctly identified changes were used for analysis of response time.

\section{Error Rate}

The main effect of driving experience was significant, $F(1,36)=5.68, p<.05, \eta^{2}=0.14$, as novice drivers had a higher error rate than experienced drivers. In line with earlier studies, the main effect of safety relevance was also significant, $F(1,36)=17.63, p<.001, \eta^{2}{ }_{p}=0.33$, as relevant targets were detected more accurately than irrelevant targets. In contrast, the main effect of target size was not significant, $F(1,36)=0.02$, $n s$. As anticipated, the interaction between driving experience and safety relevance was significant, $F(1,36)=9.81, p<.01, \eta^{2}=0.21$, yet the form of the interaction was inconsistent with any of the findings of earlier studies (see Figure 2 ). There was no difference between the two groups when detecting relevant targets, $F(1,36)=$ $0.00, n s$, but experienced drivers were more accurate at detecting irrelevant targets (the average error rate was $3.74 \%$ higher in novice drivers compared to experienced drivers), $F(1,36)=8.63$, $p<.01, \eta_{p}^{2}=0.19$. Contrary to expectation, the interaction between driving experience, safety relevance and size was not significant, $F(1,36)=0.09$, ns. Analyses were also conducted with age and gender as covariates. These showed that neither age, $F(1,34)=1.53$, ns, nor gender, $F$ $(1,34)=2.90, n s$, had an effect and they did not interact with any of the experimental variables.

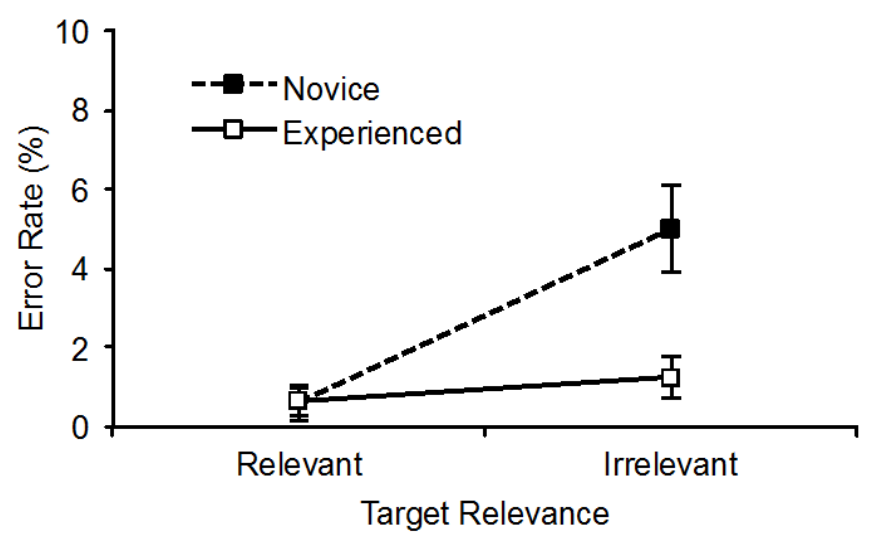

Figure 2. Error rate as a function of driving experience and target safety relevance. Error bars are \pm 1 SE

\section{Response Time}

The main effect of driving experience was not significant, $F(1,36)=0.25$, $n s$. Nevertheless, the main effect of safety relevance was significant, $F(1,36)=174.50, p<.001, \eta^{2}{ }_{p}=0.83$, with relevant targets detected faster than irrelevant targets. Moreover, the interaction between driving experience and safety relevance was significant, $F(1,36)=5.96, p<.05, \eta^{2}=0.14$. Despite the 
fact that the difference in response times between relevant and irrelevant information was slightly larger for experienced drivers than for novice drivers (experienced drivers were on average $0.01 \log _{10}(\mathrm{~ms})$ faster to detect relevant targets and $0.06 \log _{10}(\mathrm{~ms})$ slower to detect irrelevant targets than novice drivers), there was no significant difference between experienced and novice drivers for relevant, $F(1,36)=0.10$, ns, or irrelevant targets, $F(1,36)=1.53$, $n s$. Furthermore, although the main effect of size was significant (large targets were detected faster than small targets), $F(1,36)=13.26, p<.001, \eta_{p}^{2}=0.27$, there was also an interaction between safety relevance and size, $F(1,36)=12.49, p<.01, \eta^{2}=0.26$ (see Figure 3). Large relevant targets were detected faster than small relevant targets $(t(37)=4.81, p<.05, d=0.65)$ but large and small irrelevant targets were detected similarly $(t(37)=0.19, n s)$. The interaction between driving experience, safety relevance and size was borderline significant, $F(1,36)=4.06$, $n s$.

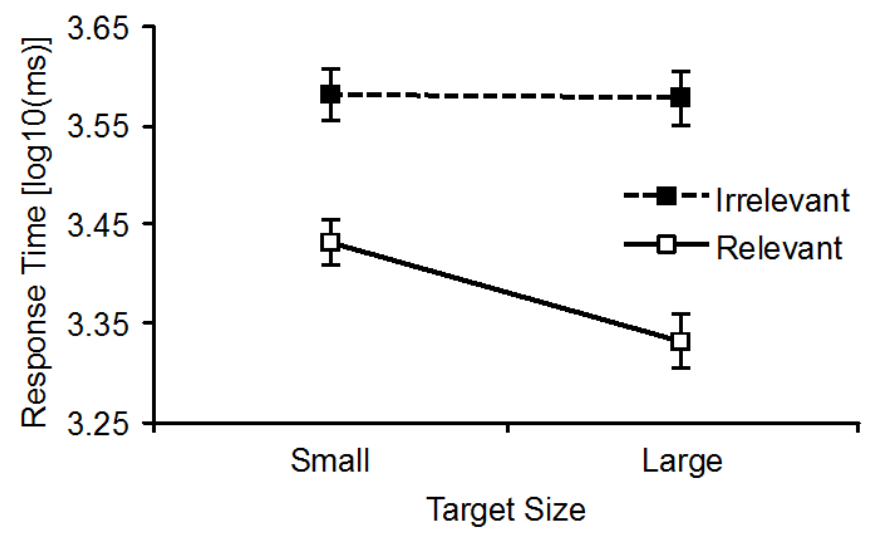

Figure 3. Response time (with $\log _{10}$ transformation) for correct change detection as a function of target safety relevance and size. Error bars are $\pm 1 \mathrm{SE}$

Analyses were also conducted with age and gender as covariates. There was no main effect of age on response time, $F(1,34)=0.02, n s$, and although there was an interaction between age, safety relevance and size, $F(1,34)=9.84, p<.01, \eta_{p}^{2}=0.22$, age did not significantly correlate with any of the within-subject conditions. Gender had a significant effect on response time, $F$ (1, 34) $=8.84, p<.01, \eta_{p}^{2}=0.21$, and male observers tended to be faster than females. Although gender did not interact with any of the experimental variables, given that there were more male participants in the experienced condition than in the novice condition, the interaction between driving experience and safety relevance on response time ought to be interpreted with caution.

\section{DISCUSSION}

Consistent with earlier research, our data suggest that all observers tend to select relevant information better than irrelevant information; however, driving experience seems to affect how accurately observers select irrelevant information. Experienced drivers were more accurate than novice drivers at detecting irrelevant information, yet there was no difference between the two groups in their ability to detect relevant information. This suggests that novice drivers might not be as efficient as experienced drivers when searching road traffic scenes for relevant information and, consequently, they have fewer resources left over to detect irrelevant information. 
The ability to detect relevant information appears to be influenced by size, but observers detected irrelevant information similarly regardless of size. It is possible that relevant information is given priority for processing and it undergoes further analysis in which secondary information, such as visual conspicuity, is used to determine its relative importance. This could explain why large relevant targets were detected faster than small ones. Irrelevant information appears to be downgraded for processing and does not undergo the secondary processing. Alternatively, the fact that relevant targets were inherently animate whereas irrelevant targets were not might have affected how observers selected information based on visual conspicuity. Passenger vehicles may have been detected faster than small road users because they are more conspicuous due to their size, typically higher speeds, and generally more predictable behaviour. In contrast, small and large irrelevant targets may have been detected similarly because they do not possess those behaviourally meaningful characteristics that road users have from a driver's perspective.

In basic research it is easy to manipulate target size, but in the real world some objects are inherently smaller than others. Moreover, some objects are simply less common on the road than others. In North America small road users are not as common as passenger vehicles which makes it difficult to disentangle the effects of familiarity and size. Therefore, another interpretation of the interaction between safety relevance and size could be that passenger vehicles are detected faster because observers are more familiar with them in the driving environment. Even so, the purpose of our study was to address some of the methodological limitations of earlier research as well as introduce the concept of manipulating visual conspicuity through target size in the driving context. In fact, we had anticipated an effect of familiarity based on driving experience, particularly for the detection of small road users. That we did not find an effect of experience on the interaction between target safety relevance and size suggests that perhaps the traditional flicker change detection task is not sensitive enough to detect those effects, if they exist. Perhaps video-based stimuli or a modified flicker task (where trials have a shorter time limit to increase the challenge of the task and the error rate) are required to elicit such an interaction. On the other hand, perhaps road user experience itself ought to be examined. Wulf, Hancock, and Rahimi (1989) suggested that the type of road user experience drivers have affects how well they detect other road users (e.g., drivers who are also motorcyclists tend to have fewer collisions with other motorcyclists compared to drivers who have no motorcycling experience). Nevertheless, this study showed effects of driving experience without appreciable differences in age, which suggests that even a small difference in years of experience can affect selection strategies.

At first glance the ability to select irrelevant information may appear immaterial to driving; however, the capacity to select extraneous information may give experienced drivers an advantage in the detection of unanticipated events or in the ability to multitask. Given that all drivers demonstrated sensitivity for object size when selecting safety-related information, future studies should consider assessing hazard anticipation training programs (e.g., Taylor et al., 2011) to determine whether it is possible to teach drivers to select small road users more efficiently.

\section{ACKNOWLEDGEMENTS}

Auto21 (Teen and Novice Driver Network), the Natural Sciences and Engineering Council of Canada, the Canadian Foundation for Innovation, and the Ontario Innovation Trust funded this research by grants to the second author. 


\section{REFERENCES}

Batchelder, S., Rizzo, M., Vanderleest, R., \& Vecera, S. (2003). Traffic scene related change blindness in older drivers. Proceedings of the Second International Driving Symposium on Human Factors in Driving Assessment, Training, and Vehicle Design, Park City, UT, 177181.

Engel, F. L. (1977). Visual conspicuity, visual search and fixation tendencies of the eye. Vision Research, 17(1), 95-108.

Enns, J. T., \& Rensink, R. (1992). VScope ${ }^{\mathrm{TM}}$ : Vision testing software for the Macintosh. Vancouver, Canada: Micropsych Software.

Galpin, A., Underwood, G., \& Crundall, D. (2009). Change blindness in driving scenes. Transportation Research Part F, 12(2), 179-185.

Groff, L. S., \& Chaparro, A. (2003). Effects of experience and task relevance on the ability to detect changes in a real-world task. Proceedings of the Human Factors and Ergonomics Society $47^{\text {th }}$ Annual Meeting, 47, 1605-1609.

Hurt, H. H. Jr., Ouellet, J. V., \& Thom, D. R. (1981). Motorcycle accident cause factors and identification of countermeasures (NTIS No. DOT-HS-5-01160). Los Angeles: University of Southern California.

Pringle, H. L., Irwin, D. E., Kramer, A. F., \& Atchley, P. (2001). The role of attentional breadth in perceptual change detection. Psychonomic Bulletin and Review, 8(1), 89-95.

Rensink, R. A., O’Regan, J. K., \& Clark, J. J. (1997). To see or not to see: The need for attention to perceive changes in scenes. Psychological Science, 8(5), 368-373.

Tanaka, M., Ikeda, M., Qingwei, G., \& Ishiguchi, A. (2009). Change-detection in driving scene images: A comparative analysis of the eye movements of experienced and novice drivers [Abstract]. Perception, 38, ECVP Abstract Supplement, 168.

Taylor, T., Masserang, K., Pradhan, A., Divekar, G., Samuel, S., Muttart, J., ... \& Fisher, D. (2011). Long-term effects of hazard anticipation training on novice drivers measured on the open road. Proceedings of the Sixth International Driving Symposium on Human Factors in Driving Assessment, Training, and Vehicle Design, Lake Tahoe, CA, 187-194.

Thornley, S. J., Woodward, A., Langley, J. D., Ameratunga, S. N., \& Rodgers, A. (2008). Conspicuity and bicycle crashes: Preliminary findings of the Taupo bicycle study. Injury Prevention, 14, 11-18.

Trick, L. M., Enns, J. T., Mills, J., \& Vavrik, J. (2004). Paying attention behind the wheel: A framework for studying the role of attention in driving. Theoretical Issues in Ergonomics Science, 5(5), 385-424.

Wulf, G., Hancock, P. A., \& Rahimi, M. (1989). Some causes of automobile-motorcycle collisions. Proceedings of the Human Factors Society 33rd Annual Meeting, 33, 910-914.

Velichkovsky, B. M., Dornhoefer, S. M., Kopf, M., Helmert, J., \& Joos, M. (2002). Change detection and occlusion modes in road-traffic scenarios. Transportation Research Part F, 5, 99-109. 